\title{
The prognostic value of cortical magnetic stimulation in acute middle cerebral artery infarction compared to other parameters
}

\author{
Th.P.J. Timmerhuis ${ }^{a}, *$ G. Hageman ${ }^{b}$, S.J. Oosterloo ${ }^{c}$, A.R. Rozeboom ${ }^{\text {d }}$ \\ ${ }^{8}$ Department of Neurology, St. Elisabeth Hospital, Hilvarenbeekseweg 60, 5022 GC Tilburg, Netherlands \\ ${ }^{b}$ Department of Neurology, Hospital Medisch Spectrum Twente, Haaksbergerstraat 55, 7500 KA Enschede, Netherlands \\ ${ }^{\mathrm{c}}$ Department of Educational Measurement and Data-analysis, University of Twente, 7500 AE Enschede, Netherlands \\ ${ }^{\mathrm{d}}$ Department of Radiology, Hospital Medisch Spectrum Twente, Haaksbergerstraat 55, 7500 KA Enschede, Netherlands
}

Received 1 December 1995; revised 20 May 1996; accepted 20 May 1996

\begin{abstract}
The prognostic value of magnetic evoked potentials (MEP), somatosensory evoked potentials (SSEP), age and radiological parameters was determined in 50 patients with acute middle cerebral artery infarction. We performed MEP and SSEP within 4 days and after 6 weeks and 3 months of the infarction and assessed clinical improvement by using the Barthel index (BI) and the Rankin scale. The localization and extent of the infarction was investigated by CT scanning or NMR. All parameters were correlated to clinical outcome and the prognostic significance of each parameter in addition to BI was determined. MEP, SSEP, and age were valuable prognostic parameters in predicting stroke outcome when used together with the BI. However, in stepwise regression analysis using all parameters simultaneously, only MEP and age significantly contributed to clinical outcome in addition to BI. Patients showed a better outcome when their MEP was normal or delayed, measured within 4 days of the infarction, compared to patients with absent MEP. Clinical outcome was better at a younger age.
\end{abstract}

Keywords: MEP; Stroke; SSEP; Prognosis; Transcranial magnetic stimulation

\section{Introduction}

Despite progress in knowledge and management of stroke, it remains difficult to predict the neurological outcome in individual stroke patients. This can be of importance with regard to clinical and rehabilitative programs and information to patients and clinicians. In the past, some studies demonstrated that clinical parameters, such as deviated conjugate eye gaze, urinary incontinence, impaired consciousness, and leg weakness have some predictive value in stroke [1-3]. Although a quantified scaled neurologic examination might give some prognostic information, one cannot make reliable predictions about functional outcome in individual patients [4].

Neurophysiological studies of stroke thus far have

*Corresponding author. Tel.: (31) (013) 5392552; Fax: (31) (013) 5350355 . investigated somatosensory evoked potentials (SSEP) and transcranial motor evoked potentials [5-8]. However, conclusions were controversial. Although an absent SSEP may predict a poor prognosis, less can be said about normal SSEPs [9]. Abnormalities of SSEP are also found in pure motor stroke [10]. Magnetic evoked potentials (MEP) could provide more accurate and prognostic information because the function of the impaired motor pathways determines neurological morbidity in stroke. Although some authors studied the value of MEP in stroke, interpretation of the results is difficult because of differences in stroke localization (infratentorial or supratentorial), time of investigation, single versus multiple infarcts, and duration of followup $[6,7,11]$.

We performed a longitudinal prospective study of 50 patients with middle cerebral artery infarction and determined the neurological outcome related to MEP, SSEP, age and radiological parameters. Because current 
neurological functioning [as assessed by the Barthel index (BI)] is a predictor of outcome, we studied the additional predictive value of MEP, SSEP, age, and radiological characteristics. More specifically, the following questions were addressed: (1) Which of the variables MEP, SSEP, age or radiological parameters has 'supplementary' predictive value if used in combination with the Barthel index? (2) Which combination of predictors can be used to predict neurological functioning?

\section{Materials and methods}

In the period March 1992 to May 1994, we investigated 64 patients with clinical evidence of a first infarction in the territory of the middle cerebral artery and with obvious neurological disability after 3 days. They had been admitted within $24 \mathrm{~h}$ after onset of symptoms. The neurological handicap was scored according to the Rankin scale and BI at day 3 or 4, after 6 weeks, and after 3 months [12,13]. For inclusion, the CT-scan or NMR had to show a single infarct in the middle cerebral artery territory after 4 days; scans were repeated after 3 months to exclude new infarctions.

Localization was scored as 'cortical', 'subcortical', or both. The extent of infarction was measured at the maximal radius and was scored 'less than $1 \mathrm{~cm}$ ', 'more than 1 but less than $5 \mathrm{~cm}$ ', 'more than 5 but less than $10 \mathrm{~cm}$ ', and 'more than $10 \mathrm{~cm}$ '. Of the 64 patients, 14 were excluded: 9 patients died before the second examination, 2 refused further cooperation, and 3 patients showed more than one infarct at second radiological examination.

We therefore studied 50 patients, 31 women and 19 men, with a mean age of $68.2(32-96)$ years. Thirteen patients were not available for examination after 3 months because of refusal (5), death (2), or logistic reasons. Thus, the data for 37 patients were available for analysis after 3 months.

Four channels were used to record average scalp SSEPs, i.e. Erb to $\mathrm{Fz}, \mathrm{C} 5$ to $\mathrm{Fz}, \mathrm{C}^{\prime} 4$ to $\mathrm{A} 2$ (A1) and C'3 to A2 (A1) with a Nicolet Pathfinder system. SSEPs were recorded on day 3 or 4 , after 6 weeks, and after 3 months and were scored 'normal', 'delayed', or 'absent'.

Magnetic motor stimulation was performed using a Medicor Magstim 200 magnetic stimulator with a 70 $\mathrm{mm}$ coil for cortical stimulation. For cervical stimulation a twin coil was used. Muscle responses were recorded with a EMG Nicolet Viking recording system with surface electrodes taped over the abductor digiti quinti muscle. Stimuli without facilitation were given with increasing intensity (stepwise $40-100 \%$ output) until an action potential in the contralateral muscles was obtained. For each measurement of MEP latency, at least three MEPs were recorded, and the shortest one was taken. MEP was considered absent if no response could be obtained with stimulation at $100 \%$ output. The computed central conduction time, i.e. the difference in conduction time after cortical and cervical stimulation, was compared to normal values [14]. MEP was scored as 'normal' (12.0-15.0 ms), 'delayed' (more than 15.0 $\mathrm{ms}$ ), or 'absent'; ipsilateral responses were registered when present. The variables MEP and SSEP were dichotomized $(0=$ 'absent' and $1=$ 'delayed' or 'normal'), because preliminary statistical analysis suggested that this would lead to the best prediction of BI. Patients were excluded if they had a history of craniotomy, epilepsy, cardial prosthetic valve, pacemaker implantation, or neurophysiological evidence of a polyneuropathy or cervical radiculopathy. All patients underwent rehabilitative therapy. Patients had to give informed consent and the study was approved by the Hospital Ethics Committee.

Parametric statistical methods were used to analyze the data. We used the general linear regression model with $\mathrm{BI}$ as a dependent variable.

\section{Results}

\subsection{Localization}

Twenty-six of the 50 infarcts were right-sided and 24 left-sided, with a cortical localization in 9 patients, a subcortical localization in 19 , and both localizations in 20 patients.

\subsection{Magnetic evoked potentials}

Thirty-two patients had an absent MEP at entry $\left(T_{1}\right)$. Their mean BI was $3.6(0-13)$ and increased to $6.3(0$ 19) at $T_{2}$ (6 weeks). Eight patients had a delayed MEP with clinical improvement on BI from $5.5(1-12)$ to 14.6 (2-20). The BI of the other 10 patients who had a normal motor response, increased from $4.9(0-10)$ at $T_{1}$ to 15.5 (4-20) at $T_{2}$ (Fig. 1).

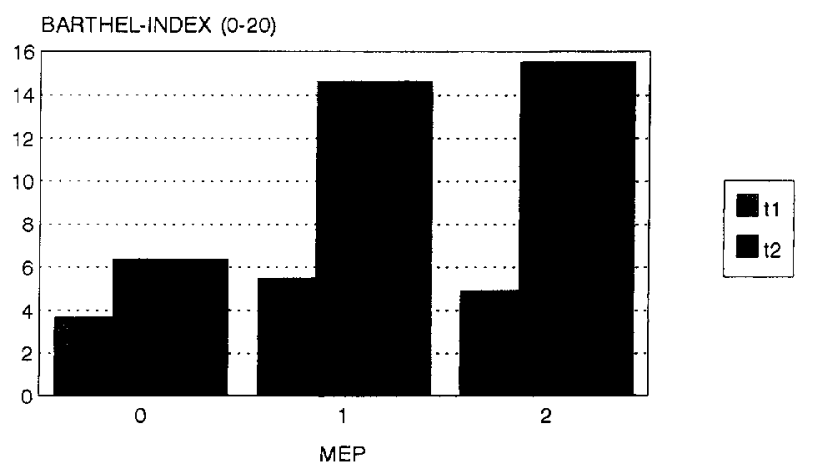

Fig. 1. Improvement of the Barthel index from $T_{1}$ to $T_{2}$ in relation to MEP. 
Table 1

Means, standard deviations and number of observations for the main variables [for MEP and SSEP: $0=$ absent, $1=$ delayed or normal; the mean is equal to the proportion $p$ of delayed or normal reactions, the standard deviation equals $\sqrt{ }(p(1-p))]$

\begin{tabular}{|c|c|c|c|c|c|c|c|c|c|}
\hline \multirow[t]{2}{*}{ Variable } & \multicolumn{3}{|l|}{$T_{1}$} & \multicolumn{3}{|l|}{$T_{2}$} & \multicolumn{3}{|l|}{$T_{3}$} \\
\hline & $M$ & S.D. & $N$ & $M$ & S.D. & $N$ & $M$ & S.D. & $N$ \\
\hline Barthel & 4.16 & 3.36 & 50 & 9.58 & 6.70 & 50 & 12.65 & 6.26 & 37 \\
\hline MEP & 0.36 & 0.48 & 50 & 0.48 & 0.50 & 50 & 0.54 & 0.51 & 37 \\
\hline SSEP & 0.43 & 0.50 & 50 & 0.60 & 0.49 & 50 & 0.74 & 0.45 & 37 \\
\hline Age & 68.54 & 13.54 & 50 & & & & & & \\
\hline
\end{tabular}

$M=$ mean; S.D. = standard deviation; $N=$ number of observations.

\subsection{Somatosensory evoked potentials}

Twenty-six patients had an absent SSEP at $T_{1}$. Their BI increased from $2.8(0-9)$ at $T_{1}$ to $6.8(0-20)$ at $T_{2}$. Five patients who had a delayed SSEP showed an improvement in BI from $7.4(4-13)$ to $10.6(4-19)$, whereas in 19 patients with a normal SSEP the BI increased from $5.2(0-12)$ to $13.1(2-20)$.

Six of the 19 patients with subcortical infarcts had delayed MEPs (32\%) as compared to 2 of the 29 patients with infarcts with cortical involvement $(7 \%)$.

The correlation coefficient for BI at different points in time was $0.59\left(T_{1}-T_{2}\right), 0.56\left(T_{1}-T_{3}\right)$ and $0.91\left(T_{2}-\right.$ $T_{3}$ ). The correlation coefficient for the Rankin scale scores at different points was $0.17,0.06$, and 0.46 , respectively. Apparently the relation between neurological function at different times as detected by BI was not reflected in the Rankin scores. Furthermore, regression analysis showed that MEP, SSEP and age could not predict the Rankin score. For this reason we present the analysis for BI only.

In none of the regression analyses were the radiological parameters (extent or localization of the lesion) predictive of the BI. Means and standard deviations of the remaining variables are shown in Table 1.

Over time the percentage of patients showing a (delayed or normal) reaction to cortical magnetic stimulation increased from $36 \%$, via 48 to $54 \%$. The percentage of patients showing a reaction to SSEP also increased, as did the mean BI.

\subsection{Prediction from time 1 to time 2}

In order to determine which of the variables predic- tors MEP, SSEP, or age had 'supplementary' predictive value (in addition to Barthel $T_{1}$ ) three regression analyses were done with the Barthel $T_{2}$ score as an dependent variable and the Barthel $T_{1}$ score and one of the variables MEP, SSEP or age as predictors.

Table 2 shows that each of the variables had, when added to the Barthel $T_{1}$ score, a regression-coefficient that was significantly different from zero. The increase in the proportion of 'explained variance' was small for SSEP (0.05), where as the contribution of age (increase in explained variance $=0.18$ ) and especially of MEP (increase in explained variance $=0.26$ ) in predicting the Barthel $T_{2}$ score was much more substantial.

The predicted BI at $T_{2}$ of two patients with the same score at $T_{1}$ differed by 7.17 points if one of the patients did not show a reaction to magnetic motor stimulation at $T_{1}$ and the other showed a (normal or delayed) reaction. The presence of an SSEP increased the predicted BI by only 3.00 points. For a given BI at $T_{1}$, the predicted Barthel score after 6 weeks decreased by 0.21 points as the age of the patient increased a year. In order to see which combination of variables at $T_{1}$ best predicted the Barthel $T_{2}$ score, we performed a stepwise regression with the Barthel $T_{1}$, SSEP, MEP, and age as potential predictors. This analysis showed that the 'best' prediction was obtained by using the Barthel $T_{1}$ score, MEP, and age as predictors. With these predictors included in the regression equation, the contribution of SSEP was no longer statistically significant $(t=0.46, p>0.30)$. The regression weights of the variables that appeared to contribute to the prediction of the Barthel $T_{2}$ score, are given in Table 3.

The proportion of variance explained by SSEP, MEP, and age was high (0.73). As a measure of the

Table 2

Regression weights (standard errors; one-sided significant probability) if the Barthel $T_{2}$ score is predicted on the basis Barthel $T_{1}$ score and one other predictor and the corresponding 'proportion explained variance' ( $\mathrm{R}$-squared). If the Barthel $T_{1}$ score is the only predictor, the 'proportion explained variance' was $0.34(n=50)$

\begin{tabular}{llcl}
\hline Second predictor & Weight Barthel $T_{1}$ & Weight second predictor & R-squared \\
\hline MEP & $0.93(0.19 ; 0.0000)$ & $7.17(1.31 ; 0.0000)$ & 0.77 \\
SSEP & $1.01(0.25 ; 0.0001)$ & $3.00(1.67 ; 0.0390)$ & 0.62 \\
Age & $1.00(0.21 ; 0.0000)$ & $-0.21(0.05 ; 0.0001)$ & 0.71 \\
\hline
\end{tabular}


Table 3

The Barthel $T_{2}$ score predicted by three variables: regression weights, standard errors, $t$-statistic, significant probability $(F(3,46)=35.47$, $p=0.0000, \mathbf{R}$-squared $=0.73$ ) and decrease in $\mathrm{R}$-squared if variable deleted

\begin{tabular}{lrlrll}
\hline Predictor & Weight & S.E. & \multicolumn{1}{l}{$T$} & $p$ & $\begin{array}{l}\text { Decrease in } \\
\text { R-squared }\end{array}$ \\
\hline MEP & 6.63 & 1.10 & 6.04 & 0.0000 & 0.21 \\
Barthel $T_{1}$ & 0.80 & 0.16 & 5.03 & 0.0000 & 0.15 \\
Age & -0.18 & 0.04 & -4.69 & 0.0000 & 0.13 \\
(Constant) & 16.50 & & & & \\
\hline
\end{tabular}

contribution of each of the three variables to this prediction, we computed the decrease in explained variance, obtained by deleting a variable (Table 3). This showed the contribution of MEP to be more important than that of SSEP or age.

\subsection{Prediction from time 1 to time 3}

Again, we first studied the 'supplementary predictive value' of each of the variables MEP, SSEP, and age separately (Table 4). SSEP did not contribute significantly to predict the Barthel $T_{3}$ score, when BI, as measured at $T_{1}$ was included as a predictor. The 'supplementary predictive value' of MEP was significant, although less impressive than it was in predicting the Barthel $T_{2}$ score. Because measurements 3 months after stroke were not available for all patients, Tables 4 and 5 are based on 37 patients only. As might be expected, prediction of the Barthel $T_{3}$ score on the basis of measurements taken a few days after stroke was less successful than the prediction of the Barthel $T_{2}$ score: the "proportion of the variability of the predicted variable explained by the predictors" (that is, the squared multiple correlation coefficient) decreased from 0.73 to 0.63 .

\subsection{Prediction from time 2 to time 3}

The correlation between the Barthel $T_{2}$ and Barthel $T_{3}$ scores was very high: $r=0.90(n=37)$. This could indicate that the level of neurological function of the patients had already stabilized after 6 weeks. As a conse- quence, prediction of the Barthel $T_{3}$ score was not improved by adding more predictors.

In fact, the regression coefficients of the other predictors, if added to the predictor Barthel $T_{2}$ score, did not differ significantly from zero.

\section{Discussion}

This study is the first in which the value of magnetic cortical evoked potentials is compared to that of other clinical, neurophysiological and radiological parameters simultaneously in purely supratentorial infarcts. Although some authors have evaluated the role of MEP in stroke, they considered infarcts and hemorrhages without comparison or prognostic statements. Stimulation was performed without facilitation because not all patients who have had a major stroke are able to induce a muscle contraction. On the other hand, scalp representation of a given muscle is significantly enlarged during facilitation [15].

We studied the outcome after 6 weeks and 3 months because earlier reports concluded that major improvement after stroke can be detected after one month [16]. This conclusion was confirmed in our study; none of the parameters, including MEP, provided additional information more valuable than the BI after 6 weeks (Barthel $\left.T_{2}\right)$. However, it can be of importance to predict the neurological improvement in the acute stage, in which case clinical and neurophysiological parameters are of value.

BI was a good predictor of neurological outcome, but we wanted to determine whether the other variables had additional prognostic value. Multiple regression analysis showed that after BI, MEP had the best predictive capacity, followed by age. Compared to these predictors SSEP did not provide additional information.

It is remarkable that the patients who died in the first weeks had normal, delayed and absent MEPs. This stresses the importance of considering both clinical and neurophysiological findings in acute stroke, as these nine patients had a low BI at study entry and their mean age was 78 years (70-85).

Gott et al., in their report on SSEP in stroke, did not find an additional value for SSEP, but they also con-

Table 4

Regression weights (standard errors; one-sided significant probability) if the Barthel $T_{3}$ score is predicted on the basis of the Barthel $T_{1}$ score and one other predictor and the corresponding 'proportion explained variance' (R-squared). If the Barthel $T_{1}$ score is the only predictor, the 'proportion explained variance' was $0.32(n=37)$

\begin{tabular}{llcl}
\hline Second predictor & Weight Barthel $T_{1}$ & Weight second predictor & R-squared \\
\hline MEP & $0.86(0.23 ; 0.0003)$ & $4.86(1.64 ; 0.0028)$ & 0.46 \\
SSEP & $0.94(0.27 ; 0.0007)$ & $0.92(1.88 ; 0.3144)$ & 0.32 \\
Age & $0.87(0.21 ; 0.0001)$ & $10.23(0.06 ; 0.0002)$ & 0.54 \\
\hline
\end{tabular}


Table 5

The Barthel $T_{3}$ score predicted by three variables: regression weights, standard errors, $t$-statistic, sign. prob. $(F(3,32)=16.71, \mathrm{R}$ squared $=0.63)$ and decrease in $\mathrm{R}$-squared if variable deleted $(n=37)$

\begin{tabular}{lrrrrr}
\hline Predictor & Weight & S.E. & $T$ & $p$ & $\begin{array}{l}\text { Decrease in } \\
\text { R-squared }\end{array}$ \\
\hline MEP & 3.95 & 1.39 & 2.84 & 0.0037 & 0.09 \\
Barthel $T_{1}$ & 0.78 & 0.19 & 4.09 & 0.0002 & 0.19 \\
Age & -0.20 & 0.05 & -3.91 & 0.0002 & 0.18 \\
(Constant) & 21.09 & & & & \\
\hline
\end{tabular}

sidered 21 transient ischaemic attacks out of 70 stroke patients in their study [4].

Macdonell et al. conclucted two studies with fewer than 20 patients and found MEP to be more valuable than SSEP in predicting stroke outcome $[11,17]$. Although they observed that SSEP changes paralleled neurological improvement, they could not prove that SSEP had prognostic value, because 6 of 9 patients who showed substantial recovery had an initial absent SSEP.

Chu et al. conducted a comparable study of 28 patients with supratentorial infarcts in the anterior and medial cerebral territory [9]. They made sum scores for MEP in the arm and leg and also found MEP to have predictive value when present.

There was a tendency for SSEP to be a negative predictor when absent. However, the mean age of subjects in their study was 55.2, substantially younger than our subjects and very young for a stroke population.

In a recent extensive study on central motor conduction time following stroke, Heald et al. studied 118 firstever stroke patients with either infarct or hemorrhage of any cerebral localization $[18,19]$. They found the highest mortality in the group with an absent MEP, with a total mortality of $36 \%$, probably because of relatively many hemorrhages. Although $16 \%$ of our patients died, some of thern had an MEP whereas others did not. It seems logical therefore to assess both clinical (BI, age, concomitant disease) and neurophysiological methods when making a prediction about the outcome for individual patients.

On the basis of these studies, it seems that MEP shows other characteristics in hemorrhages than in infarcts, which might be explained by the space occupying effect.

The stroke patients we investigated had comparable neuro-anatomical conditions. From our results we conclude that, next to a clinical disability scale like the Barthel index, cortical magnetic evoked potential and age are valuable prognostic parameters in acute middle cerebral artery infarction. Although SSEP alone provides some prognostic information it is of less value than BI, MEP or age. MEP should be recorded within a few days after the onset of stroke. In later stages neurological function as assessed by BI is the best indicator for outcome after 3 months.

\section{References}

[1] Chambers, B.R., Norris, J.W., Shurvell, B.L. and Hachinski, V.C. (1987) Prognosis of acute stroke. Neurology, 37: 221-225.

[2] Gladman, J.R.F., Harwood, D.M.J. and Barer, D.H. (1992) Predicting the outcome of acute stroke: prospective evaluation of five multivariate models and comparison with simple methods. J. Neurol. Neurosurg. Psychiatry, 55: 347-351.

[3] Allen, C.M.C. (1984) Predicting the outcome of acute strkoe: a prognostic score. J. Neurol. Neurosurg. Psychiatry, 47: 475-480.

[4] Gott, P.S., Karnaze, D.S. and Fisher, M. (1990) Assessment of median nerve somatosensory evoked potential in cerebral ischaemia. Stroke, 21: 1167-1171.

[5] Berardelli, A., Inghilleri, M., Cruccu, G., Mercuri, B. and Manfredi, M. (1991) Electrical and magnetic transcranial stimulation in patients with corticospinal damage due to stroke or motor neuron disease. Electroencephalogr. Clin. Neurophysiol., 81: 389-396.

[6] Homberg, V., Stephan, K.M. and Netz, J. (1991) Transcranial stimulation of motor cortex in upper motor neuron syndrome: its relation to the motor deficit. Electroencephalogr. Clin. Neurophysiol., 81: 377-388.

[7] Ferbert, A., Vielhaber, S., Meincke, U. and Buchner, H. (1992) Transcranial magnetic stimulation in pontine infarction: correlation to degree of paresis. J. Neurol. Neurosurg. Psychiatry, 55: $294-299$.

[8] Labar, D., Petty, G., Emerson, R., Pedley, T. and Mohr, J.P. (1991) Median nerve somatosensory evoked potentials in patients with lacunar and other small subcortical strokes. J. Neurol. Sci., 101: 221-226.

[9] Chu, N. and Wu, T. (1992) Motor response patterns and prognostic value of transcranial magnetic stimulation in stroke patients. In: M.A. Lissens (Ed.), Clinical Applications of Magnetic Transcranial Stimulation. Peeters, Leuven, pp. 127-145.

[10] Kovala, T., Tolonen, U. and Pyhtinen, J. (1991) A prospective one year follow-up study with somatosensory potentials evoked by stimulation of the posterior tibial nerve in patients with supratentorial cerebral infarction. Electroencephalogr. Clin. Neurophysiol. 80: 262-275.

[11] Macdonell, R.A.L., Donnan, G.A. and Bladin, P.F. (1989) A comparison of somatosensory evoked and motor evoked potentials in stroke. Ann. Neurol., 25: 68-73.

[12] Mahoney, F.I. and Barthel, D.W. (1965) Functional evaluation: the Barthel index. Maryland State Med. J., 14: 61-65.

[13] Rankin, J. (1957) Cerebral vascular incidents in patients over the age of 60: II. Prognosis. Scottish Med. J., 2: 200-215.

[14] Hageman, G., Eertman-Meyer, C.J. and Tel-Hampsink, J. (1993) De klinisch-diagnostische betekenis van magnetostimulatie-onderzoek. Ned. Tijdschr. Geneeskd., 137: 2323-2328.

[15] Rossini, P.M., Barker, A.T., Berardelli, A., Caramia, M.D., Caruso, G., Cracco, R.Q., Dimitrijevic, Hallet, M., Katayama, Y., Lücking, C.H., Maertens de Noordhout, A.L., Marsden, C.D., Murray, N.M.F., Rothwell, J.C., Swash, M. and Tomberg, C. (1994) Non-invasive electrical and magnetic stimulation of the brain, spinal cord and roots: basic principles and procedures for routine clinical application. Report of an IFCN committee. Electroencephalogr. Clin. Neurophysiol. 91: 79-92.

[16] Duncan, P.W., Goldstein, L.B., Matchar, D., Divine, G.W. and Feussner, J. (1992) Measurement of motor recovery after stroke; outcome assessment and sample size requirements. Stroke, 23: 1084-1089.

[17] Macdonnel1, R.A.L., Donnan, G.A. and Bladin, P.F. (1991) Se- 
rial changes in somatosensory evoked potentials following cerebral infarction. Electroencephalogr. Clin. Neurophysiol., 80: 276-283.

[18] Heald, A., Bates, D., Cartlidge, N.E.F., French, J.M. and Miller, S. (1993) Longitudinal study of central motor conduction time following stroke; natural history of central motor conduction. Brain, 116: 1355-1370.
[19] Heald, A., Bates, D., Cartlidge, N.E.F., French, J.M. and Miller, S. (1993) Longitudinal study of central motor conduction time following stroke; central motor conduction measured within $72 \mathrm{~h}$ after stroke as a predictor of functional outcome at 12 months. Brain, 116: 1371-1385. 